ARTICLE

\title{
Profiling protein expression in circulating tumour cells using microfluidic western blotting
}

Elly Sinkala1, Elodie Sollier-Christen ${ }^{2,3}$, Corinne Renier ${ }^{2,3}$, Elisabet Rosàs-Canyelles ${ }^{1,4}$, James Che ${ }^{2,5}$, Kyra Heirich ${ }^{3}$, Todd A. Duncombe ${ }^{1,4}$, Julea Vlassakis ${ }^{1,4}$, Kevin A. Yamauchi ${ }^{1,4}$, Haiyan Huang ${ }^{6}$, Stefanie S. Jeffrey ${ }^{3}$ \& Amy E. Herr ${ }^{1,4}$

Circulating tumour cells (CTCs) are rare tumour cells found in the circulatory system of certain cancer patients. The clinical and functional significance of CTCs is still under investigation. Protein profiling of CTCs would complement the recent advances in enumeration, transcriptomic and genomic characterization of these rare cells and help define their characteristics. Here we describe a microfluidic western blot for an eight-plex protein panel for individual CTCs derived from estrogen receptor-positive (ER + ) breast cancer patients. The precision handling and analysis reveals a capacity to assay sparingly available patient-derived CTCs, a biophysical CTC phenotype more lysis-resistant than breast cancer cell lines, a capacity to report protein expression on a per CTC basis and two statistically distinct GAPDH subpopulations within the patient-derived CTCs. Targeted single-CTC proteomics with the capacity for archivable, multiplexed protein analysis offers a unique, complementary taxonomy for understanding CTC biology and ascertaining clinical impact.

\footnotetext{
${ }^{1}$ Department of Bioengineering, University of California, Berkeley, 308B Stanley Hall, MC 1762, Berkeley, California 94720, USA. ${ }^{2}$ Vortex Biosciences, Inc., Menlo Park, California 94025, USA. ${ }^{3}$ Department of Surgery, Stanford University School of Medicine, Stanford, California 94305, USA. ${ }^{4}$ The UC BerkeleyUCSF Graduate Program in Bioengineering, University of California, Berkeley, California 94720, USA. ${ }^{5}$ Department of Bioengineering, University of California, Los Angeles, Los Angeles, California 90095, USA. ${ }^{6}$ Department of Statistics, University of California, Berkeley, California 94720, USA. Correspondence and requests for materials should be addressed to A.E.H. (email: aeh@berkeley.edu).
} 
T he role of circulating tumour cells (CTCs) in cancer progression is still under investigation. CTCs are rare cells that shed from a tumour into circulation at an occurrence of 1-500 cells per $7.5 \mathrm{ml}$ of blood ${ }^{1}$. Consequently, substantial research has focused on the isolation of CTCs by exploiting distinctive characteristics of these cancer cells (for example, surface protein expression, size and deformability $)^{2-5}$. High CTC counts are associated with reduced survival rates ${ }^{6}$ and low responsiveness to therapies $^{7}$. In addition, characterization of CTCs by nextgeneration sequencing has identified discordance in the gene expression between CTCs and their primary tumours ${ }^{8-10}$. These studies suggest that distinct CTC sub-populations may exist and contribute to metastasis. Nevertheless, although CTC enumeration and genomics provide insight, neither measurement fully describes phenotype. In fact, recent studies show weak correlation between genomics/transcriptomics and protein expression in some instances $^{11-14}$.

Yet, unlike single-cell genomics and transcriptomics, advances in single-cell protein assays are lagging. Strikingly, most singlecell protein assays (for unmodified endogenous targets) are single-stage immunoassays, including enzyme-linked immunosorbent assays (with direct or sandwich readout) and immunocytochemistry, as well as newer immunoassay formats designed to improve multiplexing using spatial barcoding ${ }^{15,16}$ or mass cytometry ${ }^{17}$. CTC protein analyses primarily focus on surface and secreted proteins ${ }^{18,19}$. Although important, the capability to multiplex and assay a wide range of protein targets (including intracellular signalling pathways) has been limited ${ }^{20}$. Direct measurement of multiple proteins in single-CTCs comprises a critical complement to single-CTC transcriptomic and genomic studies, as well as enumeration.

Nevertheless, target detection by single-stage immunoassays remains constrained by the specificity and availability of immunoreagents. These limitations stymie understanding of CTC phenotype in two crucial aspects. First, single-stage immunoassays have difficulty with multiplexed measurements of surface and intracellular proteins for each single cell ${ }^{21}$. Immunoassays are the de facto standard for solid tumour and CTC classification (that is, $\mathrm{CK}+, \mathrm{EpCAM}+$ and $\mathrm{CD} 45-$ expression). Yet, clinical immunoassays (for example, immunohistochemistry) are limited to $\sim 5$ proteins due to spectral imaging limitations with conventional filter sets 22,23 and difficulty in 'de-staining' cells (removing antibody probes). Flow cytometry also suffers from multiplexing shortcomings, especially with intracellular protein targets. Even more importantly, neither flow cytometry nor mass cytometry can assay small numbers of CTCs, owing to cell handling losses and dead volumes ${ }^{24}$. Second, immunoassays cannot uniquely detect a protein if a high specificity probe is unavailable. This is of particular importance in cancer, as isoform expression is increasingly implicated in patient outcome $e^{25}$ and key isoforms do not have specific antibodies available. Although mass spectrometry can measure most protein isoforms, the analytical sensitivity is insufficient for detection of key signalling proteins with single-cell resolution ${ }^{26}$.

For decades, researchers have addressed single-stage immunoassay specificity limitations by prepending an upstream polyacrylamide gel electrophoresis (PAGE) protein separation to a downstream immunoassay, thus creating a two-stage assay known as western blotting. Separating proteins by molecular mass (or mobility) before the immunoassay can identify off-target, non-specific antibody binding ${ }^{27}$. Spatially resolving proteins by size first allows a single antibody probe to detect multiple, distinct protein form ${ }^{28}$. Still, the analytical sensitivity of slab-gel western blotting requires pooling of cells to achieve detectable protein concentrations, which obscures important CTC-to-CTC protein expression level variation. To surmount this gap, we recently introduced a single-cell resolution western blot $^{29}$ optimized for study of protein expression in each of thousands of single, cultured neural stem cells ${ }^{30}$ and glioblastoma cells ${ }^{31}$. However, the current format of the single-cell western blot requires 1000s of cells to account for cell losses when settling into the microwells.

Here we introduce a rare-cell, single-cell resolution western blot $(s c \mathrm{WB})$ to measure a panel of proteins in single CTCs isolated from patients with primary estrogen receptor-positive $(\mathrm{ER}+)$ breast cancer. The rare-cell $s c \mathrm{WB}$ quantifies multiple surface and intracellular signalling proteins-in each individual CTC-allowing estimates of biological protein expression variation among CTCs, as compared with a quantitative threshold for technical variation that we establish. We show that the rare-cell $s c \mathrm{WB}$ is compatible with established CTC isolation tools, thus successfully analysing CTC populations with as few as two starting cells. In a pilot study of ER + metastatic breast cancer patient-derived CTCs, we observe a lysis-hardy CTC phenotype and the unique capacity to normalize target protein expression by the number of CTCs analysed per assay, just one CTC per $s c \mathrm{WB}$ microwell here. The rare-cell $s c \mathrm{WB}$ offers a new approach to examining CTCs, with relevance spanning from understanding CTC biology to monitoring an individual's response to therapy.

\section{Results}

Workflow enables rapid protein analysis of rare cells. We develop a microfluidic assay to measure multiple protein targets in individual CTCs. The rare-cell $s c \mathrm{WB}$ couples PAGE of singleCTC lysate with subsequent antibody probing of PAGE-resolved protein targets. The rare-cell $s c \mathrm{WB}$ assay is designed for (i) integration with existing rare-cell enrichment and isolation tools to minimize cell loss, and (ii) rapid lysis and protein separation (seconds) of isolated cell lysate to maximize local protein concentrations for detection, even with low protein copy numbers (Fig. 1). The microfluidic $s c \mathrm{WB}$ device comprises an array of $50 \mu \mathrm{m}$ diameter microwells stippled into a $60 \mu \mathrm{m}$-thin polyacrylamide (PA) gel layered on a silanized microscope slide ${ }^{29}$. Cells are seated into the microwells, lysed and protein contents are electrophoretically injected into the surrounding gel where all steps of a western blot (PAGE, blotting and probing) are performed. The integrated design of the multi-stage assay makes rapid (seconds) assay stages possible, as is needed to minimize lysate dilution by diffusion and maintain high local protein concentrations for detection.

For analysis of rare cells, we integrate the $s c \mathrm{WB}$ with a labelfree CTC collection tool that selects on cell size and deformability (Vortex chip CTC isolation technology) $^{32}$. Although the rare-cell $s c \mathrm{WB}$ is designed as agnostic to CTC collection technology, integration with a label-free cell enrichment tool allows scrutiny of cell surface receptor identity, signalling protein levels and cell variation based on characteristics that are independent of receptor expression. Our protein panel comprises targets having accepted utility in cancer subtype classification (ER, HER2 and epidermal growth factor receptor (EGFR) oncoproteins), cancer cell identification (epithelial cell adhesion molecule (EpCAM), panCK and CK8 expression), known expression in mammalian cells (glyceraldehyde 3-phosphate dehydrogenase (GAPDH) and $\beta$-tubulin) and indicative of white blood cells (WBCs; for example, high CD45, negative for EpCAM, panCK and CK8). Profiling multiple proteins aids cancer subtype classification and may inform prognosis and therapy selection in ways not otherwise apparent from primary tumour classification ${ }^{33}$. Importantly, incongruences in protein expression between the primary tumour and CTCs have been observed ${ }^{34}$, suggesting that measurement of cancer markers in both CTCs and tumours could be informative. Overexpressed intracellular, downstream targets 
i. Purify putative CTCs

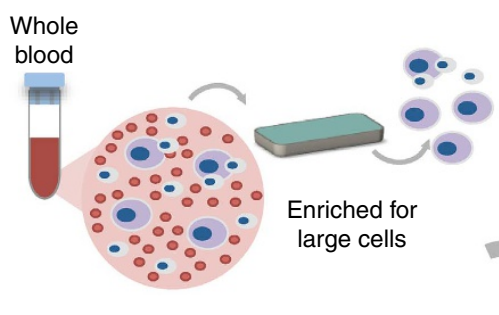

ii. Isolate a large nucleated cell into each microwell

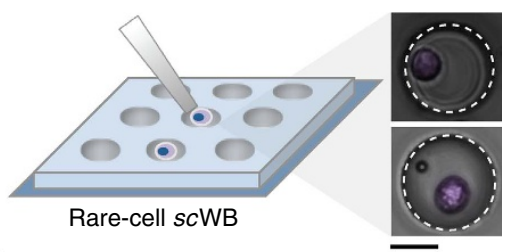

iii. Perform rare-cell scWB on each microwell
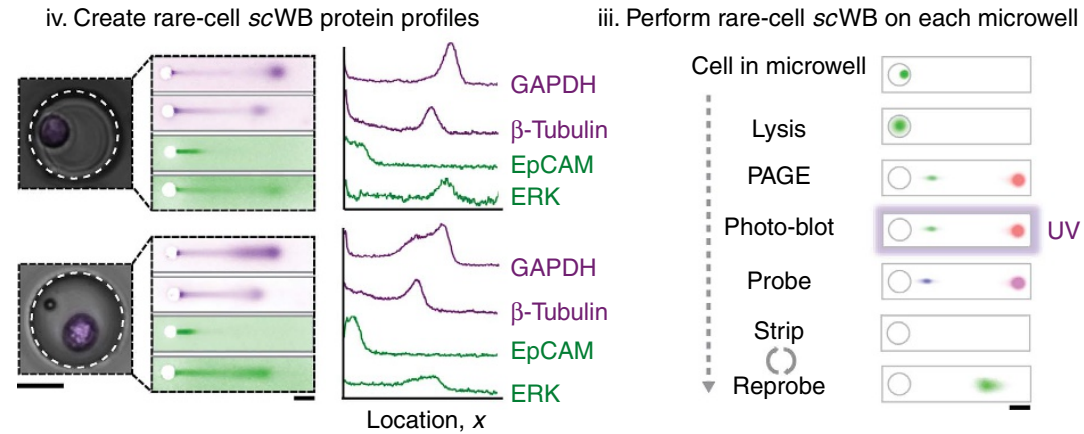

Figure 1 | Microfluidic rare-cell workflow for multiplexed western blotting of single patient-derived CTCs. Patient-derived CTCs are: Step i: enriched from 2 to $4 \mathrm{ml}$ of blood using a size- and deformability-selective microfluidic tool (Vortex HT chip) ${ }^{4}$ followed by Step ii: cell-enriched effluent ( $300 \mu \mathrm{l}$ ) is deposited directly in a mesofluidic chamber on planar scWB device. Putative CTCs are visually identified using Hoescht 33342 nuclear stain, and each identified CTC is micropipetted (under microscopy) into a $50 \mu \mathrm{m}$ diameter microwell (micrographs in inset). Step iii: after the seating of one CTC into one microwell, single-cell western blotting proceeds as in-microwell chemical CTC lysis, single-CTC protein PAGE, covalent immobilization of proteins to the gel (photo-blotting) and in-gel immunoprobing. Step iv: single-CTC lysate is analysed by western blotting and rounds of immunoprobing support the multiplexing of 12 proteins, with expression is compared among patient-derived CTCs and to spiked cell line validation studies. Scale bars, $25 \mu \mathrm{m}$ (for the cell micrographs) and $250 \mu \mathrm{m}$ (the separation micrographs).

(that is, mammalian target of rapamycin (mTOR) ${ }^{35}$, extracellular signal-regulated kinase (ERK-1/2) (ref. 36) and eukaryotic translation initiation factor $\left.4 \mathrm{e}(\mathrm{eIF} 4 \mathrm{E})^{37}\right)$ were also assayed by $s c W B$. Inhibition of intracellular signalling is an active subject of clinical trials ${ }^{38,39}$. Further, breast cancer cells have been found to have upregulated GAPDH expression, leading to interest in profiling of seemingly innocuous housekeeping proteins ${ }^{40}$.

To initiate the rare-cell scWB workflow, we dilute blood samples in phosphate buffer saline (PBS) and process the cells using the Vortex chip technology (Fig. 1, Step i). The $\sim 300 \mu \mathrm{l}$ sample effluent is then deposited into a $\sim 700 \mu \mathrm{l}$ mesofluidic polydimethylsiloxane (PDMS) chamber mated onto the top surface of the $s c \mathrm{WB}$ device. Once inside the mesofluidic chamber, we stain with Hoechst 33342 all enriched cells to identify large, nucleated cells using both bright-field and epifluorescence microscopy. The microtransfer of a single putative cancer cell follows previously established protocols by Hannemann et al. ${ }^{41}$ and others. Briefly, although monitoring under epifluorescence microscopy, each large nucleated cell was picked individually using a precision Eppendorf Transferman micromanipulator and transferred to a microwell at a target occupancy of one cancer cell per microwell (Fig. 1, Step ii and Supplementary Fig. 1). Strongly Hoechst-stained cells with diameters larger than an average WBC were selected to exclude freely suspended red blood cells and WBCs. Nevertheless, cells associated with the putative cancer cell could also be transferred. Co-transfer of CTCs and CTCassociated cells (clusters), which includes WBCs and other cell types, offers potential utility, as the presence of associated cells is hypothesized to have an impact on patient prognosis ${ }^{42}$.

Next, the rare-cell $s c \mathrm{WB}$ assay is initiated. Each cell is subjected to in-microwell chemical lysis $\left(15 \mathrm{~s}\right.$ at $55^{\circ} \mathrm{C}, 0.5 \% \mathrm{SDS}, 0.1 \%$ Triton X-100 and $0.25 \%$ sodium deoxycholate (Na-DOC)) and an electric potential is applied across the $s c \mathrm{WB}$ device for single-cell PAGE (25 s, $E=40 \mathrm{~V} \mathrm{~cm}^{-1}$, sieving matrix in an $8 \% \mathrm{~T}$ PA gel). After PAGE, proteins are immobilized in the gel using photoblotting ( $45 \mathrm{~s}$ ultraviolet exposure, PA copolymerized with $100 \mathrm{mM} \mathrm{N}$-[3-[(3-Benzoylphenyl)formamido]propyl] methacrylamide $)^{43}$, followed by several buffer exchanges and antibodyprobing cycles (Fig. 1, Step iii). The strong covalent immobilization of protein onto the gel, achieved by photoblotting, enables multiple in-gel re-probing rounds, here demonstrated for a diverse panel of protein targets for each CTC (Fig. 1, Step iv).

Rare-cell $s c W B$ validation for CTC-specific protein profile. To perform assay development and validation, we utilized healthy donor blood spiked with cancer cell lines representing three major and diverse breast cancer subtypes: triple-negative (BT-20), ER+ (MCF7) and HER2 + (SK-BR-3). To create cell suspensions, we released plated cells using trypsin and spiked 300-600 cells from one cell line at a time into a $2 \mathrm{ml}$ vial of healthy donor blood diluted $10 \times$ in PBS. The antibody probes used bind to the cytoplasmic domains of EGFR and HER2. In accordance with literature ${ }^{44}$, our fluorescence-activated cell sorting-based validation (Supplementary Fig. 2) demonstrate negligible effect of trypsin release on the detection of surface EpCAM using the antibody probe selected here. The single-cell PAGE assay is operated under denaturing but non-reducing conditions, which retains intact EpCAM epitopes ${ }^{45}$ suitable for immunoreagent-based detection. After spiking the cancer cells into healthy blood, the samples were purified in the same manner as patient-derived CTCs.

We first sought to assess the feasibility of the rare-cell workflow. A total of 50-110 min was sufficient for breast cancer 
cell isolation from the blood $(20 \mathrm{~min})$, followed by cell selection and seating of a single putative cancer cell into each microwell (30-90 min). Given a 30-90 min duration for cell selection and transfer, we measured one-cell-to-one-microwell transfers with: $42 \pm 19$ s.d. BT-20 transfers ( $n=3$ devices), $31 \pm 6$ s.d. MCF7 transfers ( $n=3$ devices), and $39 \pm 7$ s.d. SK-BR-3 transfers $(n=3$ devices) achieved. Once microwells were populated with individual cancer cells, the $s c \mathrm{WB}$ assay proceeded as follows: inmicrowell chemical cell lysis (seconds), protein PAGE and photoblotting (immobilization; $2 \mathrm{~min}$ ), subsequent in-gel primary $(2 \mathrm{~h})$ and secondary $(1 \mathrm{~h})$ antibody probing with one wash step after each probing cycle ( $1 \mathrm{~h}$ per wash), collection of fluorescence data

a

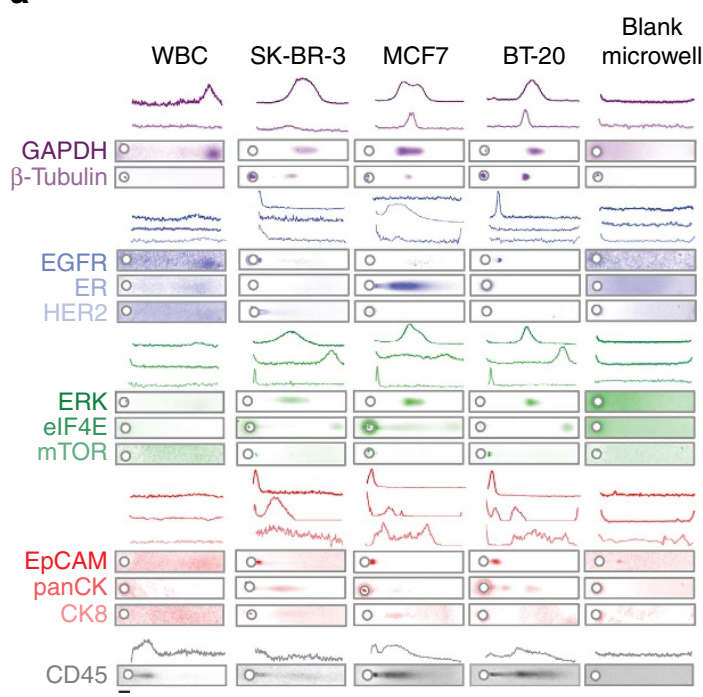

b

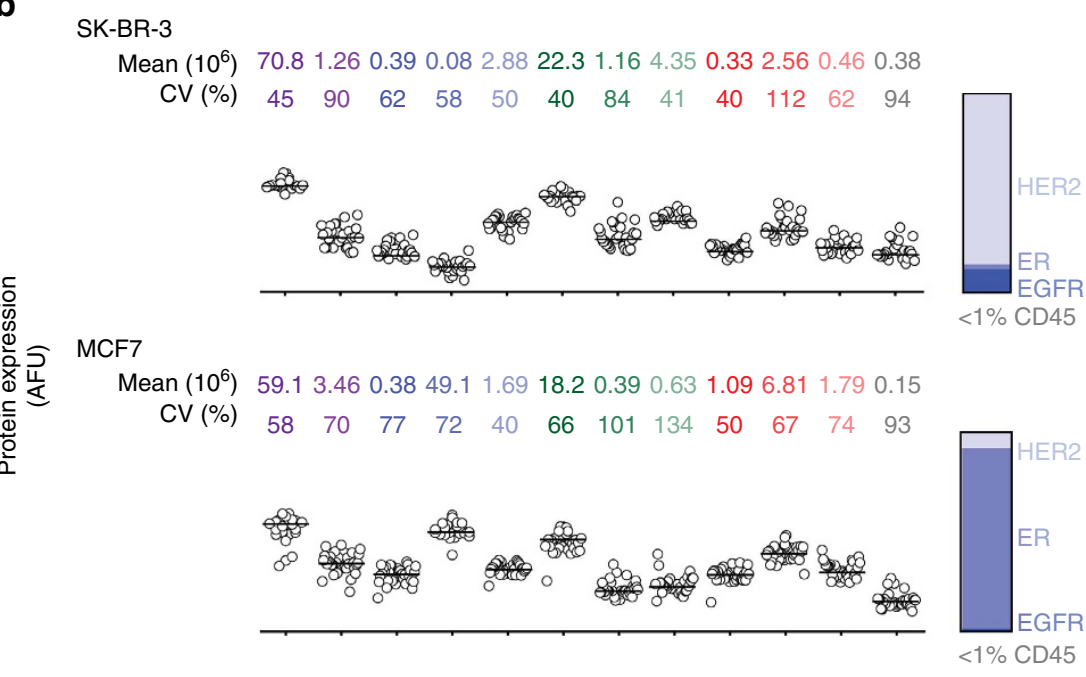

BT-20

Mean $\left(10^{6}\right) \quad 39.35 .901 .150 .080 .524 .982 .000 .261 .071 .503 .970 .16$

CV (\%) $35 \begin{array}{llllllllllll}58 & 47 & 66 & 86 & 42 & 68 & 78 & 48 & 75 & 77 & 37\end{array}$

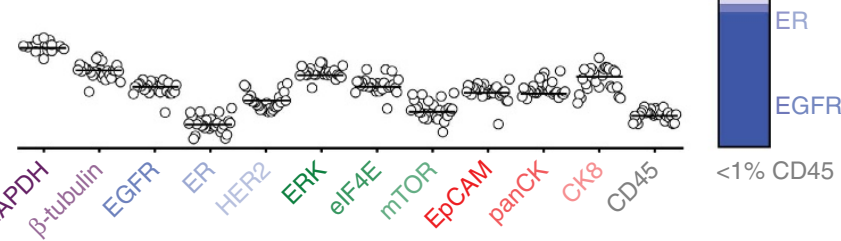

Figure 2 | The rare-cell scWB measures 12 unique protein targets in single cells from three cancer subtypes. (a) Fluorescence micrographs and intensity plots from rare-cell scWB handling and analysis of healthy blood samples, each spiked with a cancer subtype: EGFR + (BT-20), ER + (MCF7) and HER2 + (SK-BR-3). Negative controls include analysis of WBCs only and blank microwells (that is, devoid of a cancer cell). Protein panel comprises the following: control and housekeeping proteins (GAPDH and $\beta$-tubulin), oncoproteins (HER2, ER and EGFR), signalling proteins (ERK, elF4E and mTOR), common CTC classifiers (EpCAM, panCK and CK8) and a WBC indicator (CD45). Scale bar, $50 \mu \mathrm{m}$. (b) Comparative protein expression for each cancer cell (BT-20: $n=27$; SK-BR-3: $n=27$; MCF7: $n=35$ ), with mean and CV noted for each marker. Protein expression is graphed using a log-scale. Ranked oncoprotein expression for each cell line agrees with cancer subtype. Less than $1 \%$ of total protein signal is attributable to CD45. 
(45 min) and an overnight ( $12 \mathrm{~h}$ ) antibody stripping step for subsequent re-probing of additional proteins (Fig. 2a).

As baseline for gauging biological variation among patientderived CTCs, we established sources of assay technical variation. First, we considered device-to-device variability by performing technical PAGE replicates on a solution of fluorescently labeled purified protein (an ovalbumin (OVA) standard), in a manner similar to that employed in our previous $s c \mathrm{WB}$ characterization studies $^{30}$. We observed acceptably low device-to-device variation in both the protein peak width ( $w$, coefficient of variation $(\mathrm{CV})=14 \%, n=3$ devices) and electromigration distance ( $L$, peak maximum location at PAGE completion, $\mathrm{CV}=5 \%$; Supplementary Fig. 3). In corollary studies of each of the three breast cancer cell lines, we confirmed that $s c \mathrm{WB}$ measurements of protein expression were comparable between unique $s c \mathrm{WB}$ devices (Mann-Whitney $U$-test, $P>0.05$ for BT-20, SK-BR-3 and MCF7; Supplementary Information).

Second, we considered variability in protein expression measurements by benchmarking (i) epifluorescence imaging of intact, green fluorescent protein (GFP)-expressing MCF7 cells (MCF7-GFP cells) seated in microwells against (ii) $s c \mathrm{WB}$ analysis of immunoprobed GFP from those same cells (Supplementary Fig. 3). First, we observed an appreciable linear correlation between the GFP signal measured in intact MCF7-GFP cells and the corresponding immunoprobed GFP signal $\left(R^{2}=0.83\right.$, Supplementary Fig. 3). Second, we estimated the technical variation threshold that distinguishes biological from technical variation, as has been utilized in both proteomics and single-cell RNA-sequencing analyses ${ }^{46,47}$. Defining detectable biological variability in protein expression as variation that is $>3$ s.d. from the mean protein expression $\mathrm{CV}$ yields a technical variation cutoff of $32.4 \%$ (GFP-MCF7 area under the curve (AUC) mean $\mathrm{CV}+3$ s.d., where mean $\mathrm{CV}=11.0 \%$ and s.d. =7.1\%; Supplementary Fig. 3).

To establish the selectivity of the protein panel targets (Supplementary Table 1) and to assess blood matrix effects, we compared the rare-cell $s c \mathrm{WB}$ analyses of spiked cell lines with two negative controls as follows: (i) microwells occupied only by WBCs and (ii) microwells containing no cancer cells (that is, 'blank' microwells). The rare-cell $s c \mathrm{WB}$ reported positive signal for cancer markers for all spiked cell lines (BT-20, SK-BR-3 and MCF7) when a large, nucleated putative cancer cell was microtransferred into each microwell (Fig. 2a). In no instance did microscopy indicate transfer of more than one cancer cell. Further, in conjunction with microscopy, other researchers have shown microtransfer to be effective for isolation of individual cancer cells ${ }^{41,48,49}$. In negative controls, rare-cell $s c W B$ analyses of pure WBC populations reported only CD45 and GAPDH signal, with no measurable signal from any cancer-specific protein target, thus establishing co-expression of CD45 and GAPDH, and negative cancer marker response as selective for WBCs. When microwells were empty in the spiked cancer cell studies ('blank' wells), the rare-cell scWB did not detect CD45 or GAPDH. In assessing potential 'cross-talk' between proximal microwells, we did not observe detectable protein in empty microwells proximal to cancer marker-positive cell-laden microwells, with the exception of low-level EpCAM background. Thus, the validation study suggests that (i) the cancer markers are selective for putative cancer cells (as expected from the literature) and (ii) the protein panel allows the rare-cell $s c \mathrm{WB}$ to distinguish between cancer cells and WBCs.

As a corollary observation regarding possible association of WBCs with cancer cells, rare-cell scWB analysis detected CD45 signal at the level of $<1 \%$ of the total protein signal in putative cancer cells (Fig. 2b). Perhaps more surprising is the observation that the cancer cell-associated CD45 signal is not detected in 'blank' microwells proximal to cancer cell-laden microwells. The observation indicates that WBC sedimentation into neighbouring microwells does not occur often, perhaps in light of the $10^{4}$ - to $10^{5}$-fold reduction in WBCs during cancer cell isolation ${ }^{32}$. Consequently, the CD45 signal detected concomitant with the cancer panel-positive scWBs suggests the possibility of WBCs associated with the putative cancer cells and not simply dissociated (or lysed) WBCs as 'background' in the cancer cellenriched blood. Recent transcriptomic data suggests that WBCs physically associate ('cluster') with circulating tumour cells ${ }^{42}$. As is relevant to emerging clinical indicators of prognosis, the observation corroborates the utility of the rare-cell $s c \mathrm{WB}$ to capture both single CTCs and perhaps also CTCs associated with other cells (although association mechanisms are not currently understood).

Rare-cell $s c W B$ enables detailed assessment of key proteins. After establishing the selectivity of the cancer markers for cancer cells from three distinct cell lines and establishing the technical variation in the assay performance, we sought to scrutinize the known molecular classifier of each cancer subtype by rare-cell $s c \mathrm{WB}$ and compare with the literature. For each nucleated cell seated in a microwell, we ranked the relative expression of the three oncoproteins considered here (HER2, ER and EGFR; Fig. 2b). We observed concordance between the molecular classifications reported in the literature ${ }^{50-52}$ and those measured by the scWB (that is, high HER2 expression in SK-BR-3, high ER expression in MCF7 and moderate EGFR expression in BT-20; Fig. 2). Of note, the rare-cell $s c W B$ detected EGFR protein in the MCF7 cell line, in accordance with literature reports that indicate non-zero, yet low-level EGFR expression in these cells ${ }^{53}$. The primary molecular classification marker exhibited higher variability than other classification markers in two of the cancer cell lines (HER2: $\sigma_{\text {SK-BR-3 }}^{2}=2.08 \times 10^{12}, n=27$, ER: $\sigma_{\text {MCF7 }}^{2}=$ $1.24 \times 10^{15}, n=35$; EGFR: $\left.\sigma_{\text {BT- } 20}^{2}=3.15 \times 10^{11}, n=27\right)$.

For all protein panel targets across all three breast cancer cell lines (Fig. 2b), we measured variability in protein expression that exceeded the $32.4 \%$ technical variation cutoff; thus, we attribute the observed variation in protein expression to biological differences. We observed moderate-to-low variation in EpCAM expression for all cells, when compared with other proteins within each cell line (Supplementary Table 2). However, EpCAM expression in MCF7 and BT-20 cells had a wider distribution than that observed in the SK-BR-3 cell lines $\left(\sigma_{\mathrm{MCF} 7}^{2}=3.00 \times 10^{11}\right.$; $\left.\sigma_{\text {SK-BR-3 }}^{2}=1.80 \times 10^{10} ; \sigma_{\mathrm{BT}-20}^{2}=2.72 \times 10^{11}\right)$. For surface proteins known to be minimally or not expressed in each cancer subtype, we observed minimal variability in expression among each population (for example, ER in BT-20 and SK-BR-3).

Next, we applied the rare-cell scWB and Spearman's rankorder correlation to study intracellular signalling proteins across all three cell subtypes. We measured appreciable correlation between the proteins ERK and GAPDH $\left(r_{\mathrm{BT}-20}=0.759\right.$, $\left.r_{\mathrm{MCF} 7}=0.782, \quad r_{\mathrm{SK}-\mathrm{BR}-3}=0.722, \quad P<0.01\right)$, and the protein $\beta$-tubulin with both ERK $\left(r_{\mathrm{BT}-20}=0.611, r_{\mathrm{MCF} 7}=0.473, r_{\mathrm{SK}-\mathrm{BR}-3}\right.$ $=0.667, P<0.01)$ and GAPDH $\left(r_{\mathrm{BT}-20}=0.682, r_{\mathrm{MCF} 7}=0.776\right.$, $r_{\text {SK-BR-3 }}=0.825, \quad P<0.01$; Supplementary Table 3$)$. Then, within each cancer subtype, we identified specific correlations in expression levels. First, in the MCF7 cell line, we observed considerable correlation between EpCAM and ER $(r=0.670, P<0.01)$, as well as between EpCAM and panCK $(r=0.616, \quad P<0.01)$. We found panCK correlated with ER $(r=0.662, P<0.01)$. Second, in the SK-BR-3 cells, we observed higher mTOR heterogeneity than the other cell lines $\left(\sigma_{\text {SK-BR-3 }}^{2}=3.25 \times 10^{12}\right.$, as compared with $\sigma_{\mathrm{BT}-20}^{2}=5.10 \times 10^{10}$ and $\sigma_{\mathrm{MCF}}^{2}=7.65 \times 10^{11}$ ), with correlation between eIF4E and 
$\beta$-tubulin $(r=0.728, P<0.01)$, as well as panCK and eIF4E $(r=0.815, P<0.01)$. Third, BT-20 cell-to-cell expression of ERK was less variable $\left(\sigma_{\mathrm{ERK}}^{2}=4.51 \times 10^{12}\right)$ than observed in the MCF7 $\left(\sigma_{\mathrm{ERK}}^{2}=1.45 \times 10^{14}\right)$ and SK-BR-3 $\left(\sigma_{\mathrm{ERK}}^{2}=7.88 \times 10^{13}\right)$ cell lines (Supplementary Tables 2 and 3). Further, within the BT-20 population, EpCAM correlated well with eIF4E $(r=0.692$, $P<0.01)$, mTOR $(r=0.674, P<0.01)$ and ERK $(r=0.663$, $P<0.01)$. Thus, although individual cells may be categorized by the same molecular subtype, differences in downstream signalling were evident within each population. As compared with the $<5$-plex protein multiplexing that is typical in immunocytochemistry ${ }^{54}$, numerous and even unexpected relationships may be identified by deeper profiling, such as that provided by 8- to 12-plex $s c \mathrm{WB}$ analysis of cancer cells.

Lysis and electrophoresis optimization for CTC analysis. To the patient-derived CTCs, we applied lysis conditions optimized for the three cell lines (Patient $1 ; 15 \mathrm{~s}$ lysis at $55^{\circ} \mathrm{C}$ using $0.5 \%$ SDS, $0.1 \%$ Triton $\mathrm{X}-100,0.25 \%$ Na-DOC). Interestingly, we observed inadequate electrophoretic injection of CTC lysate from the microwell into the $s c \mathrm{WB}$ sieving gel, which suggested incomplete solubilization lysis and solubilization. The observation suggests differences in the lysis phenotype between the three cell lines and the patient-derived CTCs ${ }^{55}$.

Given the hardy, lysis-resistant CTC phenotype observed, we systematically varied lysis buffer composition, duration and temperature, and assessed CTC lysis and subsequent protein electrophoresis. As background, ionic detergents (SDS, Na-DOC) solubilize the cell membrane and denature proteins, whereas nonionic detergents and lysis duration enhance membrane solubilization ${ }^{56}$. Buffer temperature enhances both solubilization and denaturation. Increasing lysis duration and elevating buffer temperature present trade-offs, as increasing each factor may yield more effective cell lysis but concomitantly increase diffusive losses of single-cell lysate out of the protein-permeable microwell $^{31}$. Doubling the SDS concentration yielded satisfactory PAGE for cells from Patient 2 (Patient 2; $15 \mathrm{~s}$ lysis at $55^{\circ} \mathrm{C}$ using $1.0 \%$ SDS, $0.25 \%$ Triton X-100, $0.25 \%$ Na-DOC) by visual inspection of GAPDH injection dispersion (peak width $=303 \mu \mathrm{m}$ for 1 cell) (Fig. 3). However, CTCs derived from two subsequent patients (Patients 3 and 4) showed unacceptably high GAPDH injection dispersion, thus suggesting patient-to-patient differences in the lysis phenotype of CTCs.

Based on these findings, we further increased detergent concentrations above the critical micelle concentrations $\left(\mathrm{CMC}_{\mathrm{SDS}}=0.17 \% ; \mathrm{CMC}_{\text {Triton}-\mathrm{X}}=0.016 \%\right)$ (Pierce Protein Methods. Detergents for Cell Lysis and Protein Extraction. Protein Biology Resource Library) with Triton X-100 expected to reduce the SDS CMC, thus enhancing formation of mixed micelles that aid cell lysis and protein denaturation ${ }^{57,58}$. We extended the lysis duration and elevated the lysis buffer temperature (that is, Triton X-100 at $1 \%, \mathrm{Na}-\mathrm{DOC}$ at $0.5 \%$ and lysis buffer duration of $20-25 \mathrm{~s}$ at $60-$ $65^{\circ} \mathrm{C}$ ). The increased temperature aids in reducing the CMC. Under these stringent lysis conditions, we observed successful lysis and separations of CTCs from Patients 5, 6 and 10.

Patient-derived CTCs have a distinct biophysical phenotype. After optimization of the rare-cell $s c \mathrm{WB}$ assay on the cancer cell lines spiked into healthy blood (positive control) and WBC samples (negative control), we applied the workflow to CTCs isolated from 12 metastatic breast cancer patients. For each patient, two tubes of blood were processed, one for CTC enumeration (6 ml with 0.33 to 23.25 CTCs per $\mathrm{ml}$ ) and another for scWB (6-10 ml; Fig. 3a and Supplementary Table 4). The number of CTCs counted after immunostaining did not match to the number of CTCs analysed by rare-cell $s c \mathrm{WB}$, as each measurement was performed on a unique blood fraction with no guarantee of a matched number of CTCs in each. The rare-cell scWB was suitable for protein analysis of CTCs from patients with both low (Patient 6) and high (Patient 10) CTC counts.

Rare-cell scWB yielded a unique measurement set that established a biophysical 'lysis phenotype' for each individual CTC (Fig. 3b and Supplementary Fig. 4). Under stringent lysis conditions, we observed CTC lysis and successful electrophoretic injection of single-CTC lysate for an eight-component protein panel (Fig. 3c). Qualitatively, we observed generally low electromigration of EpCAM and ER into the PAGE gel, which we attribute to the presence of multimers (for example, $39 \mathrm{kDa}$ a

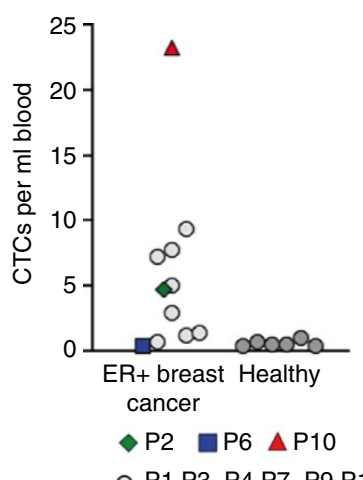

b

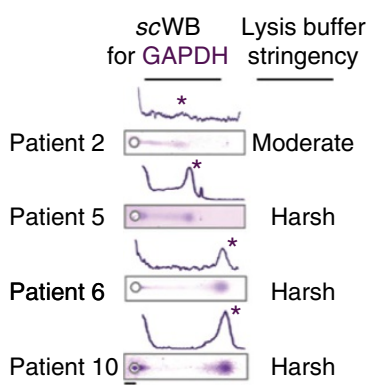

C

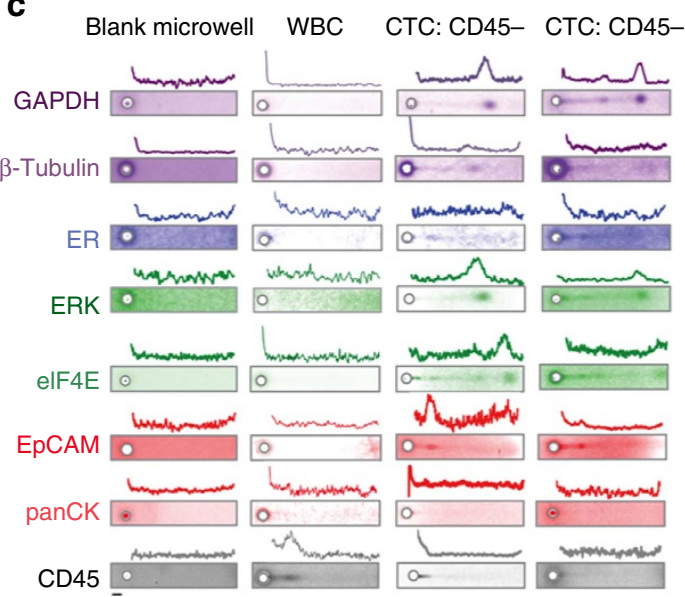

Figure 3 | Optimization of the rare-cell scWB for direct protein measurement in patient-derived CTCs. (a) CTC counts normalized to the blood volume processed by the isolation tool. CTC count for metastatic ER + breast cancer patients $(n=12)$ : 0.33-23.25 CTCs per ml; CTC count for age-matched healthy donors $(n=6)$ : 0.33-1.00 CTCs per ml. CTC threshold was set by mean $+2 \mathrm{~s}$.d. from healthy donor data at 1.06 CTCs per ml, with $81.8 \%$ of the breast cancer patients classified as positive for CTCs. Enumeration for Patient 5 was not possible, as the sample was consumed by the scWB. (b) Fluorescence micrographs and intensity plots from rare-cell scWB analysis of GAPDH in CTCs from Patients 2, 5, 6 and 10 of CTC lysis conditions. Asterisks mark GAPDH peaks. (c) Fluorescence micrographs and intensity plots from rare-cell scWB handling and analysis of representative patientderived CTCs using nomenclature from Fig. 2a. Micrographs of rare-cell scWB of patient-derived CTCs in representative cases where CD45 was not detected $($ CD45 - ). Scale bars, $50 \mu \mathrm{m}$. 
EpCAM forms dimers and tetramers ${ }^{59}$ ) and secondary/tertiary structure (for example, ER), as noted by others ${ }^{60}$. Taken together, the stringent lysis conditions were applied rare-cell $s c \mathrm{WB}$ analyses of patient-derived CTCs from Patients 5-11.

Multiplexed rare-cell $s c \mathrm{WB}$ analysis of patient-derived CTCs. Building on knowledge gained from breast cancer cell line spiking studies, we next sought to profile the protein panel in patientderived CTCs using the rare-cell $s c \mathrm{WB}$. We assayed a total of 12 unique protein targets in each putative CTC through 6 rounds of stripping and re-probing ${ }^{30}$. Of the 12 targets, we identified a subset of 8 detectable proteins in at least 1 CTC from patient samples 5, 6 and 10 (GAPDH, $\beta$-tubulin, panCK, ERK, EpCAM, ER, eIF4E and low expression CD45; Fig. 4a and Supplementary

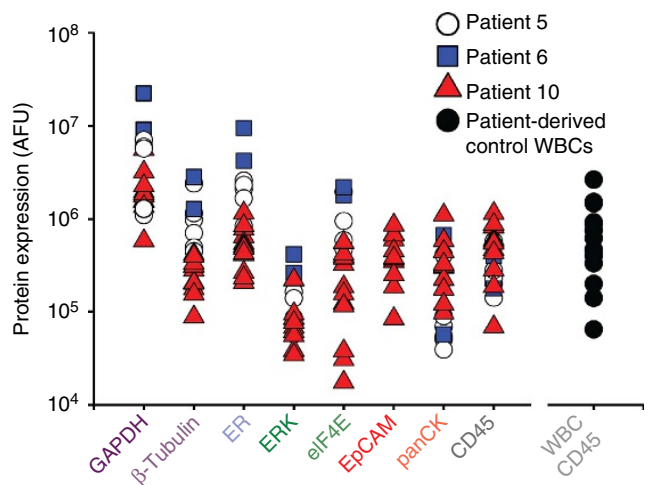

b

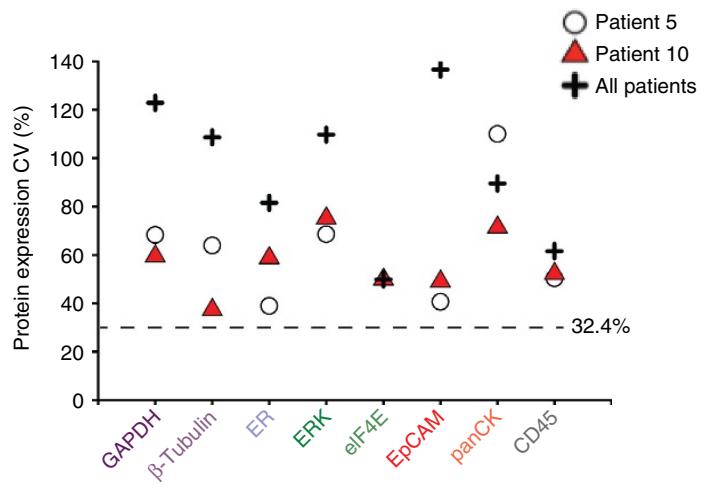

c
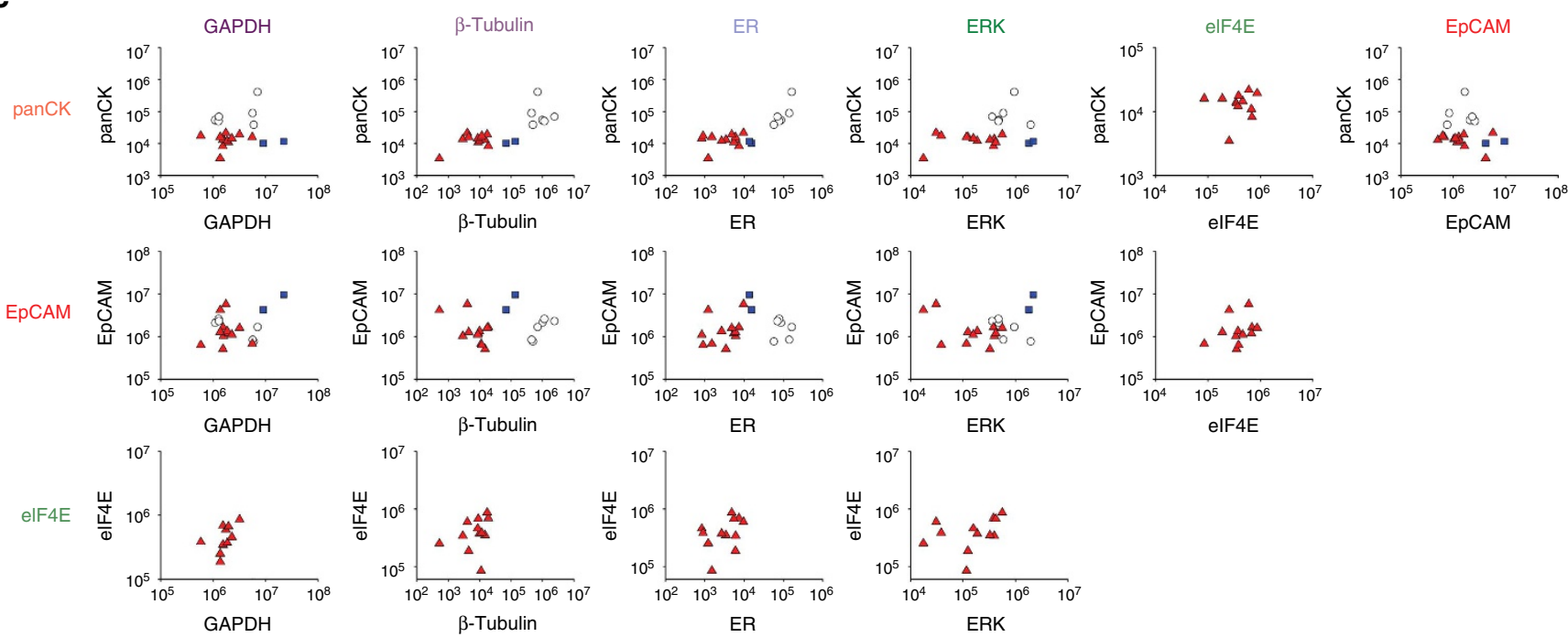

ERK
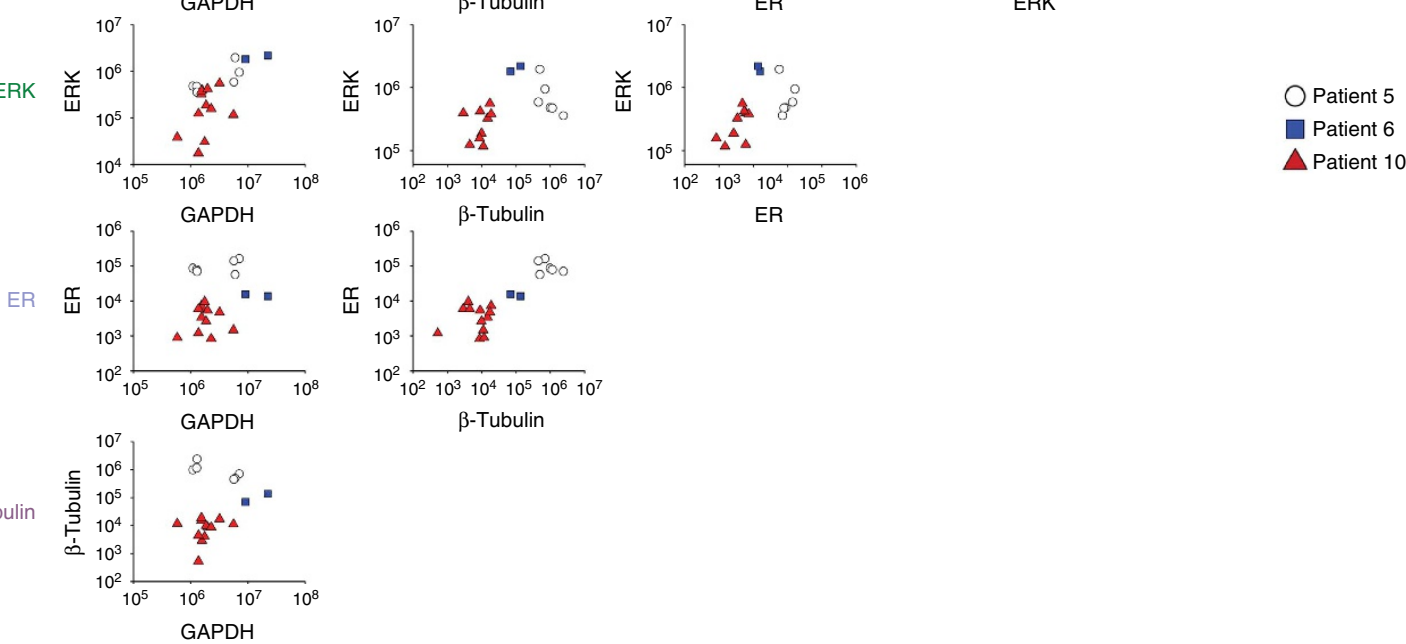

$\beta$-Tubulin

$\beta$-Tubuli

GAPDH

Figure 4 | scWB profiles for eight proteins in each individual CTC derived from three ER positive metastatic breast cancer patients. (a) Expression for each protein marker and each patient-derived CTC, with comparison with CD45 levels from scWB analyses of pure WBC controls. (b) CVs for protein expression (AUC) from the patient-derived CTCs. Dashed line indicates the threshold in protein expression variation established using GFP-expressing MCF7 cells (see Supplementary Fig. 3). (c) Biaxial plots report protein expression for all markers for each patient-derived CTC from Patients 5 ( $n=6$ ), $6(n=2)$ and $10(n=12)$. 
Fig. 5). The same protein panel was also applied to patientderived WBCs, which showed measurable signal for CD45 and GAPDH only (Supplementary Fig. 6). Twenty patient-derived cells in total were isolated using size-based enrichment followed by physical selection of only the large, nucleated cells (Hoechst 33342) aided by both bright-field and epifluorescence microscopy. All of these selected cells reported positive for the tumour markers via $s c \mathrm{WB}$. A seven-cell subset of the tumour marker-positive cells reported CD $45+$ by $s c \mathrm{WB}$, suggesting the presence of both a cancer cell and associated leukocyte(s) in $35 \%$ of cells analysed. A 13-cell subset of the tumour marker-positive cells reported CD $45-$ by $s c \mathrm{WB}$, thus strongly suggesting CTC origin alone in $65 \%$ of the cells analysed.

Protein target expression was normalized by microwell occupancy, which was one CTC per microwell with the rarecell handling workflow described. We attribute the observed CTC-to-CTC variation in protein expression levels to biological differences, as all protein panel targets had variability that exceeded the technical variation cutoff (Fig. 4b). In EpCAM expression, we observed a wider distribution among the CTCs, as compared with the ER + MCF7 cells $\left(\sigma_{\text {CTC }}^{2}=4.33 \times 10^{12}, n=20\right.$; MCF7: $\sigma_{\text {MCF7 }}^{2}=3.00 \times 10^{11}, n=35$, Levene's test: $\left.P<0.05\right)$. This CTC EpCAM expression heterogeneity underscores the challenge for immunocapture-based enrichment. In GAPDH expression, the patient-derived CTCs exhibited a larger CV than the MCF7 cells, suggesting that the patient-derived CTCs have higher variation in $\mathrm{GAPDH}\left(\mathrm{CV}_{\mathrm{CTC}}=123 \%, \mathrm{CV}_{\mathrm{MCF7}}=57.9 \%\right.$, Levene's test: $P<0.05)$. Conversely, in ER protein expression the patientderived CTCs exhibited a narrower distribution than the MCF7 cells $\left(\sigma_{\text {CTC }}^{2}=8.20 \times 10^{9}, n=20 ; \quad \sigma_{\text {MCF7 }}^{2}=1.24 \times 10^{15}\right.$, $n=35$; Levene's test: $P<0.05)$. Taken together, these findings point to potential limitations of using cancer cell lines as models for patient-derived CTCs.

To further contextualize CTC-to-CTC variation in protein expression, we compared each patient-derived CTC with all other patient-derived CTCs for Patients 5, 6 and 10 (Fig. 4c). In contrast to the cell line spiking experiments, with patient-derived CTCs we observed low variability in GAPDH expression between Patients 5, 6 and 10 (Levene's test: $P>0.05$ ). With regards to Patients 5 and 10, we observed high CVs for panCK $\left(\mathrm{CV}_{\mathrm{P} 5}=110 \% \mathrm{CV}_{\mathrm{P} 10}=71 \%, P<0.05\right)$ and $\mathrm{ERK}\left(\mathrm{CV}_{\mathrm{P} 5}=69 \%\right.$; $\left.\mathrm{CV}_{\mathrm{P} 10}=75 \%, P<0.05\right)$. In accordance with our breast cancer cell line observations, the ER + breast cancer patient-derived CTCs also exhibited correlation between ERK and GAPDH $\left(r_{\text {CTCs }}=0.579, P<0.01 ; n=20\right)$ and between the pairs ERK with $\beta$-tubulin $\left(r_{\text {СТСs }}=0.691, P<0.01 ; n=20\right)$ and EpCAM with $\beta$ tubulin $\left(r_{\mathrm{CTCs}}=0.6, P<0.01 ; n=20\right)$.

Among the patient-derived CTCs, we detected no statistically significant outlier CTCs (F-test) - with regards to exceptionally high or low protein expression-when target expression was normalized to the number of CTCs analysed in each $s c \mathrm{WB}$ (that is, one CTC analysed per microwell). However, the GAPDH expression suggests two distinct CTC sub-populations (Fig. 4c), which was confirmed with a F-test comparing a one-population model and a two-subpopulation model for the data resulting in an F-statistic of 3.89 and a $P$-value of 0.035 (degrees of freedom: $(1,4)$ ), thus suggesting two possible populations of GAPDH expression.

\section{Discussion}

The rare-cell $s c \mathrm{WB}$ expands the repertoire of available single-cell analysis tools, an area where direct measurement of multiple protein targets in each CTC is hindered by reliance on singlestage immunoassays. Expanding the number of proteins profiled in a single CTC may aid identification of CTCs that do not fit contemporary taxonomies in light of the rapidly evolving understanding of CTC biology and individual responses to therapy. Notably, both existing and emerging precision cancer therapies are targeting proteins ${ }^{35,39}$; thus, monitoring multiple upregulated proteins in blood-derived CTCs may inform therapeutic selection to maximize the benefit to each specific patient at each specific time point. Consequently, we measure eight surface and intracellular proteins with single-CTC resolution using a microfluidic targeted proteomics tool, the rare-cell $s c \mathrm{WB}$. Complementary single-cell resolution technologies such as flow cytometry and mass cytometry find analyses of such rare cells challenging, owing to sampling losses (that is, mass cytometry samples up to $\sim 30 \%$ of the initial cell population $)^{61}$. The scWB leverages short distances and timescales to rapidly complete lysis, PAGE and protein blotting of proteins in single CTCs. The careful control of sample and analysis affords the unique option to forgo normalization by protein housekeeping proteins and directly assign protein levels on a per CTC basis.

To first validate and then optimize the rare-cell $s c \mathrm{WB}$ tool, we spiked healthy blood with cells from each of three breast cancer cell lines to model three major molecular breast cancer classifications. We enriched for the spiked cancer cells and assayed via scWB. Workflow durations of $50-110 \mathrm{~min}$ are well within the timeframe of other CTC processing and analysis methods ${ }^{62}$. scWB classification of each cell line subtype agreed with expected molecular classification, but with heterogeneity in surface and intracellular protein expression detected at the singlecell level within the cells of each cell line. Tool validation studies further established the conditions needed for cell lysis, electrophoresis, blotting and probing, as well as established levels of technical variability. Rapid unit operations (lysis, electrophoresis and immobilization to the gel scaffold) and quick handling minimized dilution and diffusive losses of the cellular contents before assay completion, allowing the assay to maintain high local protein concentrations, even if local protein copy numbers are low (for example, $\sim 30,000$ molecules per cell) ${ }^{30}$.

Pilot testing of the rare-cell $s c W B$ collection-isolation-analysis workflow on blood from ER + breast cancer patients yielded CTC enumeration data paired with $s c \mathrm{WB}$ protein analyses (Supplementary Table 4). The scWB revealed a robust physical CTC phenotype resistant to the chemical lysis conditions that were optimized on three breast cancer cell lines. We attribute the hardy CTC phenotype to differences in cell type, handling and microenvironment as compared with breast cancer cell lines. For example, inclusion of fetal bovine solution (FBS) in the cell line culture medium is known to introduce a high concentration of lipids; thus, the lipid membrane composition of a cell grown in FBS would not necessarily be representative of primary cells ${ }^{63}$. Observation of the separation quality aided systematic optimization of lysis conditions suitable for a subset of eight protein targets, while minimizing protein losses. Experiments performed with our final lysis protocol suggest patient-to-patient variation in the physical CTC phenotype, thus mirroring observations even in cultured CTCs, where doubling time, point mutations and drug sensitivity vary within a CTC population $^{64}$.

The precision and detection sensitivity of the rare-cell scWB enables direct analysis of individual, patient-derived CTCs, obviating the need for post-isolation cell culture. Variation in cell state and response is proposed to be a driver of cancer progression and therapy resistance ${ }^{65}$. The rare-cell scWB identified differences between the CTC protein expression profiles, including both wider EpCAM expression ranges in CTCs (as compared with the three cell lines) and sub-populations of CTCs having statistically distinct GAPDH expression levels. Expansion of our multiplexed protein measurements with label- 
free CTC isolation to characterize additional subpopulations including cancer stem cell-like CTCs ${ }^{66}$ and CTCs undergoing $\mathrm{EMT}^{67}$ are of continuing interest. Looking forward to longitudinal studies, inclusion of protein levels in CTC phenotype could make CTC taxonomy more precise, as CTCs are thought to navigate away from the primary tumour perhaps diverging at the proteomic level ${ }^{54,68}$.

Based on our observations on CD45 expression in rare-cell $s c \mathrm{WB}$ analyses of large, nucleated cells, we see next steps developing in a manner that parallels the development of CTC enrichment tools optimized for 'CTC cluster' analyses, so that future rare-cell western blotting work is focusing on specific assay optimization for CTC clusters, as both the CTC isolation tool ${ }^{32}$ and the microtransfer protocol ${ }^{42}$ used here have been shown to be suitable for CTC cluster handling.

Important to longitudinal studies, the $s c \mathrm{WB}$ is archivable ${ }^{30}$, which allows later-date profiling of new targets of interest with previously analysed single-CTC lysates. CTC lysates are covalently immobilized to the PA gel layer on the $s c \mathrm{WB}$ device, a stable linkage compatible with long-term storage for retrospective CTC analysis. Although chemical fixation of CTCs also yields biospecimens suitable for long-term storage and retrospective studies, cell fixation is incompatible with stripping procedures and suffers from several pre-analytical variables including the following: epitope disfigurement during fixation especially changes in posttranslational modifications, loss of antigenicity owing to over- and under-fixation, and sample degradation with aging. In addition, analytical variables including target cross-reactivity with moderate specificity antibodies, lab-to-lab variation and qualitative but not quantitative analyses, all have an impact on final outcomes. Although the rare-cell $s c \mathrm{WB}$ requires further long-term storage performance characterization and larger-scale patient studies, the targeted proteomics tool introduced here presents a promising approach to multiplexed, archival protein analysis of single CTCs with direct relevance to longitudinal studies. Even longer term, the microfluidic form factor of the assay may find utility in low-resource settings, especially with further engineering integration of the cell purification and handling fluidics, as is underway.

\section{Methods}

SU-8 and PA gel fabrication. Fabrication of the SU-8 microwell mold master and PA gels was performed as described previously ${ }^{29,30}$. The cell line and CTC experiments used an $8 \% \mathrm{~T}$ PA gel, with arrays of $50 \mu \mathrm{m}$ diameter and $60 \mu \mathrm{m}$-deep microwells. All PA gels were chemically polymerized with $0.08 \%$ APS and $0.08 \%$ TEMED. A separate SU-8 master was created to fabricate the PDMS mesofluidic insert used to localize large volumes $(\sim 700 \mu \mathrm{l})$ of enriched cells over the microwell array in the PA gel layer. The insert dimensions were $37.5 \mathrm{~mm} \times 50 \mathrm{~mm}$ with an opening comprising the volume reservoir of $20 \mathrm{~mm} \times 30 \mathrm{~mm}$. PDMS polymer base and curing agent were mixed (ratio 10:1), degassed under vacuum, poured over the insert master and cured for $2 \mathrm{~h}$ at $70^{\circ} \mathrm{C}$

Cell lines. To validate the rare-cell $s c \mathrm{WB}$, we acquired healthy donor blood and spiked with cell lines representing three major breast cancer subtypes: triplenegative (BT-20), ER + (MCF7) and HER2 + (SK-BR-3). BT-20, MCF7 and SKBR-3 cells were obtained from the American Type Culture Collection and authenticated using short tandem repeat analysis (Promega). All cell lines tested negative for mycoplasma. BT-20 (ER - /PR - /HER2 - ) was maintained in Eagle's minimal essential medium supplemented with $1 \%$ penicillin/streptomycin and $10 \%$ FBS. MCF7 (ER + /PR - /HER2 - ) was maintained in RPMI 1640 supplemented with $1 \%$ penicillin/streptomycin, $0.01 \mathrm{mg} \mathrm{ml}^{-1}$ insulin (Invitrogen) and $10 \% \mathrm{FBS}$. SK-BR-3 (ER - /PR - /HER2 +) was maintained in McCoy's 5A supplemented with $1 \%$ penicillin/streptomycin and $10 \%$ FBS.

A GFP-expressing MCF7 cell line, used to determine technical variation, was obtained from the American Type Culture Collection and authenticated using short tandem repeat analysis (Promega), and tested negative for mycoplasma. The cell line was maintained in RPMI 1640 supplemented with $1 \%$ penicillin/ streptomycin, $0.01 \mathrm{mg} \mathrm{ml}^{-1}$ insulin (Invitrogen) and 10\% FBS. All cell lines were cultured in an incubator held at $37^{\circ} \mathrm{C}$ under $5 \% \mathrm{CO}_{2}$ and tested for mycoplasma contamination.

Patient recruitment and blood donation. Twelve patients with advanced breast cancer were recruited, with informed consent, according to a protocol approved by the Institutional Review Board (Stanford IRB 350-Panel 3-Protocol 5630) from the Department of Oncology at the Stanford School of Medicine. Blood was drawn in EDTA BD tubes, stored at room temperature and processed within $5 \mathrm{~h}$ after collection.

Rare-cell enrichment from blood samples. A previously reported and commercially available microfluidic tool (Vortex Biosciences, Menlo Park, CA) was used for label-free isolation of circulating cancer cells in both the cell line spiking and cancer patient blood experiments. For spiking experiments, cells were dissociated with $1.5 \mathrm{ml}$ of $0.25 \%$ trypsin (Life Technologies) and incubated in full media at room temperature to recover from exposure to trypsin. Cells were immediately spiked into healthy blood samples diluted $10 \times$ with PBS and enriched through the Vortex HT chip, which uses microscale vortices to retain large cancer cells, while allowing smaller blood cells to exit as effluent ${ }^{32}$. The microfluidic device was first primed with PBS. Then, the diluted blood sample was processed through the Vortex HT chip $\left(8 \mathrm{ml} \mathrm{min}^{-1}\right)$ followed by a wash step with PBS to remove contaminating red blood cells and WBCs $\left(8 \mathrm{ml} \mathrm{min}^{-1}\right)$. Stopping the flow dissipates the vortices and releases the cancer cells from the microscale reservoirs for direct deposition on the top surface of the $s c \mathrm{WB}$ platform. The enriched volume was $\sim 300 \mu \mathrm{l}$ and was contained by a mesofluidic PDMS insert that sits atop the $s c \mathrm{WB}$. For the spiking experiments, 300-600 cells from 1 cell line were spiked into $1 \mathrm{ml}$ healthy donor blood and processed using the Vortex chip. For patient blood experiments, the cells isolated in the vortices were directly collected into the mesofluidic PDMS insert seated on top of the $s c$ WB PA gel for cell positioning into microwells. For both cell line spiking and patient-derived cell experiments, a volume of blood was reserved for subsequent red blood cell lysis to perform control experiments with WBCs.

Preparation of WBCs. WBCs were prepared by lysing the red blood cells with Buffer EL (Qiagen). Briefly, $0.5 \mathrm{ml}$ of whole blood was combined with $2.5 \mathrm{ml}$ of Buffer EL (Qiagen). The tube was inverted several times and incubated for 10-15 $\mathrm{min}$ at room temperature. After centrifugation at $228 \mathrm{~g}$ for $5 \mathrm{~min}$ at room temperature, the supernatant was discarded. The pellet was re-suspended with $2.5 \mathrm{ml}$ of Buffer EL and the process repeated. Finally, the WBCs were washed once with $1 \mathrm{ml}$ of Buffer EL, pelleted and re-suspended in $0.5 \mathrm{ml} \mathrm{PBS}$.

scWB protocol. The $s c \mathrm{WB}$ assay comprises six steps. The $s c \mathrm{WB}$ device utilizes microwells cast into a thin layer of a photoactive PA gel seated on microscope glass slide. Once aliquoted into the mesofluidic insert, cell nuclei were stained (Hoechst 33342) to identify target cells, and a micromanipulator (Eppendorf Transferman) and aspiration (Eppendorf Cell Vario) manually positioned individual cells into each microwell. A combined lysis and electrophoresis buffer was poured directly onto the PA gel where the cells were lysed in-well and then subjected to PAGE $\left(E=40 \mathrm{~V} \mathrm{~cm}^{-1}\right)$. Lysis buffer was heated in a water bath and the temperature was recorded with a thermometer immediately before use. After the PAGE separation, proteins were immobilized in the gel via brief ultraviolet activation (Lightningcure, LC5 Hamamatsu) of benzophenone methacrylamide cross-linked into the PA gel. Immobilized proteins were probed in-gel by diffusing primary and then fluorescently labelled secondary antibody probes into the PA gel layer. A fluorescence microarray scanner (Genepix 4300A, Molecular Devices) equipped with four-laser lines (488, 532, 594 and 635) acquired fluorescence readout. Subsequent rounds of antibody stripping were performed for multiplexed protein analysis as detailed previously ${ }^{29,30}$. The $s c \mathrm{WB}$ assay can be completed within $\sim 20 \mathrm{~h}$

Antibodies. Primary antibodies and fold dilutions against GAPDH (1:20, goat polyclonal antibody (pAb); SAB2500450, Sigma), $\beta$-tubulin (1:10, rabbit pAb; ab6046, Abcam), EpCAM (1:10, rabbit pAb; 3599, Cell Signaling), EGFR (1:10, mouse monoclonal antibody; 2322, Cell Signaling), ER (1:10, rabbit monoclonal antibody; RM-9101-S0, ThermoScientific), HER2 (1:10, mouse monoclonal antibody; MA513105, Pierce), ERK1/2 (1:10, rabbit monoclonal antibody; 4695, Cell Signaling), eIF4E (1:10, rabbit monoclonal antibody; 2067, Cell Signaling), mTOR (1:10, rabbit monoclonal antibody; 2983, Cell Signaling), panCK (1:10, rabbit pAb; Z0622, Dako) and CK8 (1:10, mouse monoclonal antibody; C5301, Sigma) were the immunoprobes in both breast cancer cell lines (BT-20, MCF7 and SK-BR-3) and patient-derived CTCs. For analysis of the MCF7-GFP cell line, an anti-GFP antibody (ab6673, Abcam) followed by anti-goat AlexaFluor 555-conjugated secondary antibody (A21432, Invitrogen) were used. Secondary antibodies to goat IgG pre-labelled with AlexaFluor 488 and 555 (A11055 and A21432), mouse IgG pre-labelled with AlexaFluor 488, 555 and 647 (A21202, A31570 and A31571), and rabbit IgG pre-labelled with AlexaFluor 488, 555 and 647 (A21206, A31572 and A31573) were used as prepared by the vendor (Invitrogen). All secondary antibodies were applied as a 1:20 dilution. 
Flow cytometry. Flow cytometry analysis was performed on MCF7 cells to ascertain the effects of enzymatic detachment on the EpCAM antigen. Briefly, MCF7 cells were detached from tissue culture plates either by trypsin-EDTA (0.25\%, Gibco 25200072) or by EDTA alone (Ultrapure 0.5 M EDTA Gibco 15575020 diluted in PBS to $5 \mathrm{mM}$ ). Half the cells were labelled with anti-EpCAMAlexaFluor488 (mouse, monoclonal antibody, 53-8326-42, eBioscience) and half were labelled with mouse IgG AlexaFluor488 (A21202) as an isotype control. For labelling, $2 \times 10^{6}$ cells were resuspended in $100 \mu$ of $3 \%$ BSA (Sigma A2058) in PBS containing antibody at a concentration of $1 \mu \mathrm{g} \mathrm{ml}^{-1}$ and incubated over ice for $30 \mathrm{~min}$. Cells were washed three times in PBS, then resuspended in resuspension buffer (1\% BSA, $5 \mathrm{mM}$ EDTA in PBS) to prevent aggregation. Cells were analysed on a Guava flow cytometer (Millipore). A total of 10,000 events were collected per sample, four samples per experimental group $(n=4)$ and data were compiled and analysed using FlowJo software.

Reproducibility. To measure the run-to-run variation in PAGE performance, we assayed solutions of purified OVA protein (O34781, Thermo Fisher Scientific) pre-labelled with AlexaFluor 488 diluted in PBS to a final concentration of $1 \mu \mathrm{M}$. PA gels were incubated with $100 \mu \mathrm{l}$ of OVA solution for $1 \mathrm{~h}$, to allow partitioning of OVA into the microwells after which the $s c \mathrm{WB}$ protocol was implemented. To measure the run-to-run variation in rare-cell $s c \mathrm{WB}$ performance (including cell lysis), we performed technical replicates on two separate $s c \mathrm{WB}$ devices for two aliquots each from a suspension of each cell line (BT-20, SK-BR-3 and MCF7). Each cell suspension was pipetted on top of the $s c \mathrm{WB}$ device and gravity-settled into microwells with excess cells washed off using a solution of $1 \times$ PBS. After completing the $s c \mathrm{WB}$ protocol, GAPDH expression levels were measured (Supplementary Fig. 3); statistical equivalence of the GAPDH expression distributions between the technical replicates was tested using the Mann-Whitney $U$-test ('ranksum' function in MATLAB R2013A). Mann-Whitney $U$-test $P$-values were $0.1257,0.7578$ and 0.7815 for BT-20 $(n=59$ and 65$)$, SK-BR-3 $(n=34$ and $n=30)$ and MCF7 ( $n=42$ and 40), respectively. The null hypothesis that the GAPDH protein expression distributions are equivalent across the technical replicates was supported.

Threshold for technical variation. Using a model GFP-expressing MCF7 cell line, we compared variation in GFP expression levels obtained by (i) fluorescence imaging of whole cells with (ii) $s c \mathrm{WB}$ analysis of probed GFP from those same cells. After establishing correlation between the two modalities, we established a technical variation threshold as described in the Results section. To perform the analyses, a suspension of GFP-expressing MCF7 cells ( $\sim 1$ million cells per $\mathrm{ml}$ in $1 \times$ PBS) was pipetted onto the $s c$ WB device and cells settled by passive gravity into microwells. Excess cells were washed off with $1 \times \mathrm{PBS}$ as described elsewhere ${ }^{29,30}$.

For whole-cell imaging, epifluorescence microscopy recorded GFP fluorescence from MCF7-GFP cells seated in microwells (Olympus IX71 inverted fluorescence microscope, Andor iXon + EMCCD camera, X-cite Lumen Dynamics mercury excitation lamp, ASI motorized stage controlled in Metamorph software, Molecular Devices). Supplementary Fig. 3 reports representative whole-cell fluorescence images $(\times 10$ Olympus UPlanFLN, numerical aperture 0.45 objective, GFP filter set Chroma $49011 \mathrm{ET}$, a binning of 1 and an exposure time of $200 \mathrm{~ms}$ ). For $s c \mathrm{WB}$ analysis, the protocol described was used after imaging with MCF7-GFP cells lysed ( $15 \mathrm{~s}$ in $55^{\circ} \mathrm{C}$ RIPA-like lysis buffer $(0.5 \%$ SDS, $0.25 \% \mathrm{Na}$-DOC, $0.1 \%$ Triton X-100 in $0.5 \times$ Tris-glycine), followed by PAGE $\left(20 \mathrm{~s}^{2} 40 \mathrm{~V} \mathrm{~cm}^{-1}\right)$, photo-blotting $(45 \mathrm{~s})$, antibody probing for GFP (1:10 dilutions of anti-GFP antibody in $1 \times$ TBST with $5 \% \mathrm{BSA}, 2 \mathrm{~h}$ ), wash ( $30 \mathrm{~min}$ in $1 \times \mathrm{TBST}$ ), secondary immunoprobing (1:10 anti-goat AlexaFluor 555-conjugated secondary antibody in $1 \times$ TBST, $1 \mathrm{~h})$, wash (30 min in $1 \times \mathrm{TBST})$, rinsed in water and dried in a nitrogen stream. For whole-cell images, a fluorescence intensity profile was generated in the microwell region of interest in ImageJ and the AUC was determined. For $s c \mathrm{WB}$ peaks, the AUC for the immunoprobed GFP peak was calculated using the $s c \mathrm{WB}$ analysis protocol (Supplementary Fig. 3). Cells with similar ( $<5 \%$ variation) GFP AUC were binned and considered a homogeneous GFP-expressing sample, with a $1.27-3.37 \%$ difference in AUC from the lowest and highest GFP AUC of each bin observed. The technical variation cutoff was defined as 3 s.d. above the average $\mathrm{CV}$ of protein expression (for a $99.7 \%$ confidence interval).

Data analysis and processing. Quantification of protein PAGE and probing used in-house MATLAB scripts as described in Kang et al. ${ }^{29}$ Band widths were characterized by Gaussian curve fitting in MATLAB (R2014b, Curve Fitting Toolbox) if the Gaussian had a $R^{2}$-value $>0.7$. If $R^{2}$-value was $<0.7$ for a marker, the integrated intensity for the region of interest was calculated.

Statistical analyses. Multiple statistical analyses were performed to compare protein distribution, correlations and potential outliers. To determine significance between the different protein CVs observed, we performed a $t$-test statistic and used a permutation test to determine the $P$-values. The Levene's test was used to determine non-equivalence of the variance between the markers for each cell line (BT-20, SK-BR-3 and MCF7). To classify a group of CTCs as a sub-population based on GAPDH expression in Patient 5, an F-test for model selection was performed. Model 1 assumed one population exists and model 2 assumed two sub-populations exist. The F-test compares the two models with the null hypothesis, considering the data follows model 1 instead of model 2 . When the $P$-value is $<0.05$, the null hypothesis can be rejected.

To detect correlation in protein expression between proteins, a Spearman's rank correlation was performed, as the correlation of protein expression between two proteins was expected to be monotonic but not necessarily linear. Two proteins in the panel were sequentially paired (Supplementary Table 3 ) to determine possible correlations. Only correlations with a $P$-value $\leq 0.01$ were considered significant.

Data availability. The authors declare that all the data are available within the article file and its Supplementary Information or from the corresponding author upon reasonable request.

\section{References}

1. Miller, M. C., Doyle, G. V \& Terstappen, L. W. Significance of circulating tumor cells detected by the cellsearch system in patients with metastatic breast colorectal and prostate cancer. J. Oncol. 2010, 617421 (2009).

2. Talasaz, A. H. et al. Isolating highly enriched populations of circulating epithelial cells and other rare cells from blood using a magnetic sweeper device. Proc. Natl Acad. Sci. USA 106, 3970-3975 (2009).

3. Nagrath, S. et al. Isolation of rare circulating tumour cells in cancer patients by microchip technology. Nature 450, 1235-1239 (2007).

4. Sollier, E. et al. Size-selective collection of circulating tumor cells using Vortex technology. Lab Chip 14, 63-77 (2014).

5. Zheng, S. et al. Membrane microfilter device for selective capture, electrolysis and genomic analysis of human circulating tumor cells. J. Chromatogr. A 1162 154-161 (2007).

6. Rack, B. et al. Circulating tumor cells predict survival in early average-to-high risk breast cancer patients. J. Natl Cancer Inst. 106, dju066 (2014).

7. Sandri, M. T. et al. Changes in circulating tumor cell detection in patients with localized breast cancer before and after surgery. Ann. Surg. Oncol. 17, 1539-1545 (2010).

8. Sieuwerts, A. M. et al. mRNA and microRNA expression profiles in circulating tumor cells and primary tumors of metastatic breast cancer patients. Clin. Cancer Res. 17, 3600-3618 (2011).

9. Lohr, J. G. et al. Whole-exome sequencing of circulating tumor cells provides a window into metastatic prostate cancer. Nat. Biotechnol. 32, 479-484 (2014).

10. Deng, G. et al. Single cell mutational analysis of PIK3CA in circulating tumor cells and metastases in breast cancer reveals heterogeneity, discordance, and mutation persistence in cultured disseminated tumor cells from bone marrow. BMC Cancer 14, 456 (2014).

11. Gygi, S. P., Rochon, Y., Franza, B. R. \& Aebersold, R. Correlation between protein and mRNA abundance in yeast. Mol. Cell Biol. 19, 1720-1730 (1999).

12. Washburn, M. P. et al. Protein pathway and complex clustering of correlated mRNA and protein expression analyses in Saccharomyces cerevisiae. Proc. Natl Acad. Sci. USA 100, 3107-3112 (2003).

13. Ghaemmaghami, S. et al. Global analysis of protein expression in yeast. Nature 425, 737-741 (2003).

14. Zhang, B. et al. Proteogenomic characterization of human colon and rectal cancer. Nature 513, 382-387 (2014).

15. Fan, R. et al. Integrated barcode chips for rapid, multiplexed analysis of proteins in microliter quantities of blood. Nat. Biotechnol. 26, 1373-1378 (2008).

16. Zhang, Y. et al. Single-cell codetection of metabolic activity, intracellular functional proteins, and genetic mutations from rare circulating tumor cells. Anal. Chem. 87, 9761-9768 (2015).

17. Bendall, S. C., Nolan, G. P., Roederer, M. \& Chattopadhyay, P. K. A deep profiler's guide to cytometry. Trends Immunol. 33, 323-332 (2012).

18. Alix-Panabières, C. EPISPOT assay: detection of viable DTCs/CTCs in solid tumor patients. Recent Results Cancer Res. 195, 69-76 (2012).

19. Yao, X. et al. Functional analysis of single cells identifies a rare subset of circulating tumor cells with malignant traits. Integr. Biol. (Camb) 6, 388-398 (2014).

20. Crespo, M. et al. Androgen receptor expression in circulating tumour cells from castration-resistant prostate cancer patients treated with novel endocrine agents. Br. J. Cancer 112, 1166-1174 (2015).

21. Zbigniew, D., Robinson, J. P. \& Roederer, M. (eds). Essential Cytometry Methods (Academic Press, 2009).

22. Stack, E. C., Wang, C., Roman, K. A. \& Hoyt, C. C. Multiplexed immunohistochemistry, imaging, and quantitation: A review, with an assessment of Tyramide signal amplification, multispectral imaging and multiplex analysis. Methods 70, 46-58 (2014).

23. Harouaka, R., Kang, Z., Zheng, S.-Y. \& Cao, L. Circulating tumor cells: advances in isolation and analysis, and challenges for clinical applications. Pharmacol. Ther. 141, 209-221 (2014). 
24. Perfetto, S. P., Chattopadhyay, P. K. \& Roederer, M. Seventeen-colour flow cytometry: unravelling the immune system. Nat. Rev. Immunol. 4, 648-655 (2004).

25. Ward, T. M. et al. Truncated p110 ERBB2 induces mammary epithelial cell migration, invasion and orthotopic xenograft formation, and is associated with loss of phosphorylated STAT5. Oncogene 32, 2463-2474 (2013).

26. Yang, M., Nelson, R. \& Ros, A. Toward analysis of proteins in single cells: a quantitative approach employing isobaric tags with MALDI mass spectrometry realized with a microfluidic platform. Anal. Chem. 88, 6672-6679 (2016).

27. Saper, C. B. An open letter to our readers on the use of antibodies. J. Comp. Neurol. 493, 477-478 (2005).

28. Mahmood, T. \& Yang, P. C. Western blot: technique, theory, and trouble shooting. N. Am. J. Med. Sci. 4, 429-434 (2012).

29. Kang, C.-C. et al. Single-cell resolution western blotting. Nat. Protoc. 11, 1508-1530 (2016)

30. Hughes, A. J. et al. Single-cell western blotting. Nat. Methods 11, 749-755 (2014).

31. Kang, C.-C., Lin, J.-M. G., Xu, Z., Kumar, S. \& Herr, A. E. Single-cell western blotting after whole-cell imaging to assess cancer chemotherapeutic response. Anal. Chem. 86, 10429-10436 (2014).

32. Che, J. et al. Classification of large circulating tumor cells isolated with ultra-high throughput microfluidic Vortex technology. Oncotarget 7, 1274812760 (2016).

33. Gradilone, A. et al. Circulating tumor cells (CTCs) in metastatic breast cancer (MBC): prognosis, drug resistance and phenotypic characterization. Ann. Oncol. 22, 86-92 (2011).

34. Fehm, T. et al. Determination of HER2 status using both serum HER2 levels and circulating tumor cells in patients with recurrent breast cancer whose primary tumor was HER2 negative or of unknown HER2 status. Breast Cancer Res. 9, R74 (2007).

35. Baselga, J. Targeting the phosphoinositide-3 (PI3) kinase pathway in breast cancer. Oncologist 16, 12-19 (2011).

36. McCubrey, J. A. et al. Roles of the Raf/MEK/ERK pathway in cell growth, malignant transformation and drug resistance. Biochim. Biophys. Acta 1773, 1263-1284 (2007).

37. Anthony, B., Carter, P. \& De Benedetti, A. Overexpression of the protooncogene/translation factor $4 \mathrm{E}$ in breast-carcinoma cell lines. Int. J. Cancer 65, 858-863 (1996)

38. O'Reilly, K. E. et al. mTOR inhibition induces upstream receptor tyrosine kinase signaling and activates Akt. Cancer Res. 66, 1500-1508 (2006).

39. Janku, F. et al. PI3K/AKT/mTOR inhibitors in patients with breast and gynecologic malignancies harboring PIK3CA mutations. J. Clin. Oncol. 30, 777-782 (2012).

40. Krasnov, G. S., Dmitriev, A. A., Snezhkina, A. V \& Kudryavtseva, A. V. Deregulation of glycolysis in cancer: glyceraldehyde-3-phosphate dehydrogenase as a therapeutic target. Expert Opin. Ther. Targets 17, 681-693 (2013).

41. Hannemann, J. et al. Quantitative high-resolution genomic analysis of single cancer cells. PLoS ONE 6, e26362 (2011).

42. Aceto, N. et al. Circulating tumor cell clusters are oligoclonal precursors of breast cancer metastasis. Cell 158, 1110-1122 (2014).

43. Hughes, A. J. \& Herr, A. E. Microfluidic western blotting. Proc. Natl Acad. Sci. USA 109, 21450-21455 (2012)

44. Sukhdeo, K. et al. Multiplex flow cytometry barcoding and antibody arrays identify surface antigen profiles of primary and metastatic colon cancer cell lines. PLoS ONE 8, e53015 (2013).

45. Schnell, U., Cirulli, V. \& Giepmans, B. N. G. EpCAM: Structure and function in health and disease. Biochim. Biophys. Acta 1828, 1989-2001 (2013).

46. Molloy, M. P., Brzezinski, E. E., Hang, J., McDowell, M. T. \& VanBogelen, R. A. Overcoming technical variation and biological variation in quantitative proteomics. Proteomics 3, 1912-1919 (2003).

47. Streets, A. M. et al. Microfluidic single-cell whole-transcriptome sequencing. Proc. Natl Acad. Sci. USA 111, 7048-7053 (2014).

48. Babayan, A. et al. Heterogeneity of estrogen receptor expression in circulating tumor cells from metastatic breast cancer patients. PLOS ONE 8, e75038 (2013)

49. Dago, A. E. et al. Rapid phenotypic and genomic change in response to therapeutic pressure in prostate cancer inferred by high content analysis of single circulating tumor cells. PLoS ONE 9, e101777 (2014).

50. Subik, K. et al. The expression patterns of ER, PR, HER2, CK5/6, EGFR, KI-67 and AR by immunohistochemical analysis in breast cancer cell lines. Breast Cancer Basic Clin. Res. 4, 35-41 (2010).

51. Neve, R. M. et al. A collection of breast cancer cell lines for the study of functionally distinct cancer subtypes. Cancer Cell 10, 515-527 (2006).

52. Holliday, D. L. \& Speirs, V. Choosing the right cell line for breast cancer research. Breast Cancer Res. 13, 215 (2011).
53. Mamot, C. et al. Epidermal growth factor receptor (EGFR)-targeted immunoliposomes mediate specific and efficient drug delivery to EGFR- and EGFRvIII-overexpressing tumor cells. Cancer Res. 63, 3154-3161 (2003).

54. Cristofanilli, M. Circulating tumor cells, disease progression, and survival in metastatic breast cancer. Semin. Oncol. 33, 9-14 (2006).

55. Burdall, S. E., Hanby, A. M., Lansdown, M. R. J. \& Speirs, V. Breast cancer cell lines: friend or foe? Breast Cancer Res. 5, 89-95 (2003).

56. RIPA-SDS Buffer. Cold Spring Harb. Protoc. doi:10.1101/pdb.rec084541 (2015).

57. Sharma, R., Varade, D. \& Bahadur, P. Mixed micelles of triton x-100 and sodium dodecyl sulfate and their interaction with polymers. J. Dispers. Sci. Technol. 24, 53-61 (2003).

58. Owoyomi, O., Jide, I., Akanni, M. S., Soriyan, O. O. \& Morakinyo, M. K. Interactions between sodium dodecylsulphate and Triton X-100: molecular properties and kinetics investigations. J. Appl. Sci. 5, 729-734 (2005).

59. Balzar, M. et al. Epidermal growth factor-like repeats mediate lateral and reciprocal interactions of Ep-CAM molecules in homophilic adhesions. Mol. Cell Biol. 21, 2570-2580 (2001).

60. Kumar, R. et al. The dynamic structure of the estrogen receptor. J. Amino Acids 2011, 1-7 (2011).

61. Bandura, D. R. et al. Mass cytometry: technique for real time single cell multitarget immunoassay based on inductively coupled plasma time-of-flight mass spectrometry. Anal. Chem. 81, 6813-6822 (2009).

62. Karabacak, N. M. et al. Microfluidic, marker-free isolation of circulating tumor cells from blood samples. Nat. Protoc. 9, 694-710 (2014).

63. Francis, G. L. Albumin and mammalian cell culture: Implications for biotechnology applications. Cytotechnology 62, 1-16 (2010).

64. Yu, M. et al. Cancer therapy. Ex vivo culture of circulating breast tumor cells for individualized testing of drug susceptibility. Science 345, 216-220 (2014).

65. Meacham, C. E. \& Morrison, S. J. Tumour heterogeneity and cancer cell plasticity. Nature 501, 328-337 (2013).

66. Aktas, B. et al. Stem cell and epithelial-mesenchymal transition markers are frequently overexpressed in circulating tumor cells of metastatic breast cancer patients. Breast Cancer Res. 11, R46 (2009).

67. Armstrong, A. J. et al. Circulating tumor cells from patients with advanced prostate and breast cancer display both epithelial and mesenchymal markers. Mol. Cancer Res. 9, 997-1007 (2011).

68. Steeg, P. S. Tumor metastasis: mechanistic insights and clinical challenges. Nat Med. 12, 895-904 (2006).

\section{Acknowledgements}

We thank the breast cancer patients and healthy donors who generously consented to provide the blood samples necessary for this study, as well as the nurses, clinical coordinators (Violet Hanft, RN, BSN, OCN) and clinicians (Douglas Blayney, M.D.; Mark Pegram, M.D.; Shruti Sheth, M.D.; George Sledge, M.D.) who assisted with patien recruitment. The research reported in this publication was supported in part by the US National Institutes of Health and the National Cancer Institute (R21CA183679 to A.E.H.), the National Institute of Biomedical Imaging and Bioengineering (R21EB019880 to A.E.H.), and the Office of the Director's New Innovator program (DP2OD007294 to A.E.H.) with a Diversity Supplement (to E.S.), a NetScientific grant (to E.S.C., C.R. and S.S.J.), a CIRM Predoctoral Fellowship and Obra Social 'la Caixa' Fellowship (to E.R.C.) and a National Science Foundation Graduate Research Fellowship (to T.A.D., K.A.Y. and J.V.). UC Berkeley Biomolecular Nanotechnology Center (BNC) provided support for device fabrication.

\section{Author contributions}

E.S., E.S.C., C.R., S.S.J. and A.E.H. designed experiments. E.S. performed single-CTC western blottings and analysis. E.S.C. and C.R. performed CTC enrichment. E.S.C. and C.R. performed red blood cell lysis. J.C. performed patient CTC enumeration. E.R.C. performed fluorescence-activated cell sorting-based validation experiments, purified protein control experiments and experiments for $s c \mathrm{WB}$ benchmarking against fluorescence imaging. K.R.H. coordinated patient recruitment and healthy blood samples collection. T.A.D., K.A.Y. and J.V. designed software for fluorescence quantitation. E.S. and H.H. performed statistical analysis. All authors wrote manuscript.

\section{Additional information}

Supplementary Information accompanies this paper at http://www.nature.com/ naturecommunications

Competing financial interests: E.S., T.A.D., J.V., K.A.Y. and A.E.H. has financial interest in intellectual property related to the device and assay described here, and may benefit from royalties from licensing. Further, A.E.H. has financial interest in commercialization efforts. E.S.C., C.R. and J.C. have financial interests in Vortex Biosciences. S.S.J. has research funded by Vortex Biosciences. All other authors declare no competing financial interest. 
Reprints and permission information is available online at http://npg.nature.com/ reprintsandpermissions/

How to cite this article: Sinkala, E. et al. Profiling protein expression in circulating tumour cells using microfluidic western blotting. Nat. Commun. 8, 14622 doi: $10.1038 /$ ncomms14622 (2017).

Publisher's note: Springer Nature remains neutral with regard to jurisdictional claims in published maps and institutional affiliations. (c) (i) This work is licensed under a Creative Commons Attribution 4.0 International License. The images or other third party material in this article are included in the article's Creative Commons license, unless indicated otherwise in the credit line; if the material is not included under the Creative Commons license, users will need to obtain permission from the license holder to reproduce the material. To view a copy of this license, visit http://creativecommons.org/licenses/by/4.0/

(C) The Author(s) 2017 